\title{
Blow-Up of Solutions for a Class of Nonlinear Pseudoparabolic Equations with a Memory Term
}

\author{
Huafei $\mathrm{Di}^{1}$ and Yadong Shang ${ }^{1,2}$ \\ ${ }^{1}$ School of Mathematics and Information Science, Guangzhou University, Guangzhou, Guangdong 510006, China \\ ${ }^{2}$ Key Laboratory of Mathematics and Interdisciplinary Sciences of Guangdong Higher Education Institutes, Guangzhou University, \\ Guangzhou, Guangdong 510006, China
}

Correspondence should be addressed to Yadong Shang; gzydshang@126.com

Received 7 June 2014; Accepted 1 July 2014; Published 4 August 2014

Academic Editor: BoQing Dong

Copyright (C) 2014 H. Di and Y. Shang. This is an open access article distributed under the Creative Commons Attribution License, which permits unrestricted use, distribution, and reproduction in any medium, provided the original work is properly cited.

We consider the nonlinear pseudoparabolic equation with a memory term $u_{t}-\Delta u-\Delta u_{t}+\int_{0}^{t} \lambda(t-\tau) \Delta u(\tau) d \tau=\operatorname{div}\left(|\nabla u|^{p-2} u\right)+$ $u^{1+\alpha}, x \in \Omega, t>0$, with an initial condition and Dirichlet boundary condition. Under negative initial energy and suitable conditions on $p, \alpha$, and the relaxation function $\lambda(t)$, we prove a finite-time blow-up result by using the concavity method.

\section{Introduction}

In this paper, we consider the initial boundary value problem for a class of nonlinear pseudoparabolic equations with a memory term:

$$
\begin{gathered}
u_{t}-\beta \Delta u-\gamma \Delta u_{t}+\int_{0}^{t} \lambda(t-\tau) \Delta u(x, \tau) d \tau \\
=\delta \operatorname{div}\left(|\nabla u|^{p-2} \nabla u\right)+f(u), \quad x \in \Omega, t>0, \\
u(x, 0)=u_{0}(x), \quad x \in \Omega, \\
\left.u(x, t)\right|_{\partial \Omega}=0, \quad x \in \partial \Omega, t>0,
\end{gathered}
$$

where $\Omega \subset \mathrm{R}^{n}$ is a bounded domain with a smooth boundary $\partial \Omega, \lambda: \mathrm{R}_{+} \rightarrow \mathrm{R}$ is a given continuous function, $\beta, \gamma$, and $\delta$ are all real constant parameters, $p>2$, and $\operatorname{div}\left(|\nabla u|^{p-2} \nabla u\right)$ is the so-called $p$-Laplace operator. This type of equations describes a variety of important physical processes, such as the analysis of heat conduction in materials with memory, electric signals in nonlinear telegraph line with nonlinear damping, viscous flow in materials with memory [1], vibration of nonlinear elastic rod with viscosity [2], nonlinear bidirectional shallow water waves [3], and the velocity evolution of ion-acoustic waves in a collisionless plasma when an ion viscosity is invoked [4].
Equation (1) includes many important mathematical physics models.

In the absence of the memory term, the viscous term, and $p$-Laplace operator term $(\gamma=\delta=0, \lambda(s)=0), \beta=1$, the model reduces to semilinear parabolic equation:

$$
u_{t}-\Delta u=f(u), \quad x \in \Omega, t>0 .
$$

On the existence, nonexistence, and the properties of solutions of (2), there have been many results [5-9].

In the absence of the memory term and $p$-Laplace operator term $(\delta=0, \lambda(s)=0), \beta=\gamma=1$, the model reduces to semilinear pseudoparabolic equation:

$$
u_{t}-\Delta u-\Delta u_{t}=f(u), \quad x \in \Omega, t>0 .
$$

Kaikina et al. [10] discussed the periodic boundary value problem of (3) under some assumption forms of nonlinear function $f$. Cao et al. [11] investigated a class of periodic problems of pseudoparabolic type equations with nonlinear periodic sources. A rather complete classification of the exponent $p$ was given, in terms of the existence and nonexistence of nontrivial and nonnegative periodic solutions. Cao et al. [12] dealt with the Cauchy problem for semilinear pseudoparabolic equations. Existenceand uniqueness of local 
solutions were proved, and the large-time behavior was investigated. Kaikina [13] and $\mathrm{Xu}$ and $\mathrm{Su}$ [14] discussed the initial boundary value problems of pseudoparabolic equation (3) under some classes of nonlinear function $f(u)$. They obtained some sufficient conditions of existence and uniqueness of local solutions and the large-time behavior of global solutions.

In the absence of the memory term and the viscous term $(\lambda(s)=0, \gamma=0), \beta=0, \delta=1$, (1) becomes nonlinear parabolic equation with $p$-Laplace nonlinear term:

$$
u_{t}=\operatorname{div}\left(|\nabla u|^{p-2} \nabla u\right)+f(u), \quad x \in \Omega, t>0 .
$$

Tsutsumi [15] studied the initial boundary value problem of (4) with $f(u)=u^{1+\alpha}$, where $p<2+\alpha$. He obtained the existence of global weak solutions by using the potential well method. Liu and Zhao [16] considered the same problem with critical initial conditions $J\left(u_{0}\right)=d$ or $I\left(u_{0}\right)=0$ and proved the existence of global solution for this problem. Xu et al. [17] discussed (4) at the high energy level, where $p<$ $2+\alpha<\infty$ if $n \leq p$ and $p<2+\alpha \leq n p /(n-p)$ if $n>p$. They proved the finite time blow-up of solutions by the comparison principle and variational methods. Messaoudi in [18] considers an initial boundary value problem related to (4) and proves, under suitable conditions on $f$, a blow-up result for solutions with vanishing or negative initial energy.

In the absence of the viscous term and $p$-Laplace operator $(\gamma=0, \delta=0), \beta=0$, Gripenberg [19] considered the nonlinear parabolic equation with Volterra integral term equation:

$$
\begin{array}{r}
u_{t}=\int_{0}^{t} k(t-s) \sigma\left(u_{x}(x, s)\right)_{x} d s+f(x, t), \\
x \in(0,1), \quad t \geq 0 .
\end{array}
$$

He investigated the initial boundary value problem of (5) and established the global existence of a strong solution of the problem.

In the absence of the viscous term and $p$-Laplace operator $(\gamma=0, \delta=0)$, as $\beta=1$, the model reduces to the equation

$$
u_{t}-\Delta u=\int_{0}^{t} b(t-\tau) \Delta u(\tau) d \tau+f(u), \quad x \in \Omega, t>0 .
$$

Yin [20] obtained the global existence of a classical solution of (7) under the assumption of a one-sided growth condition. Messaoudi [21] investigated a semilinear parabolic equation with the viscoelastic memory term. He established the finite time blow-up result for the solution with negative or vanishing initial energy for nonlinear function $f(u)=|u|^{p-2} u$.

To the best of our knowledge, there are few works on the study of nonlinear pseudoparabolic equation with memory term of Volterra integral type. Shang and Guo [22-24] investigated the initial boundary value problem and initial value problem of the nonlinear pseudoparabolic equations with Volterra integral term:

$$
\begin{aligned}
& u_{t}-f(u)_{x x}-u_{x x t} \\
& \quad+\int_{0}^{t} \lambda(t-s) \sigma\left(u(x, s), u_{x}(x, s)\right)_{x} d s \\
& =f\left(x, t, u, u_{x}\right), \quad x \in(0,1), \quad t>0 .
\end{aligned}
$$

They proved the existence, uniqueness, and regularities of the global strong solution and gave some conditions of the nonexistence of global solution. In 2007, Ptashnyk [25] investigated the initial boundary value of degenerate quasilinear pseudoparabolic equations with memory term. He obtained some existence results of global solutions. Up to now, there are not any research works on the multidimensional nonlinear pseudoparabolic equations with memory term.

In the present work, we deal with the initial boundary problem of the nonlinear pseudoparabolic equation with the memory term of Volterra integral type, the damping term, and $p$-Laplace operator:

$$
\begin{gathered}
u_{t}-\Delta u-\Delta u_{t}+\int_{0}^{t} \lambda(t-\tau) \Delta u(\tau) d \tau \\
=\operatorname{div}\left(|\nabla u|^{p-2} \nabla u\right)+u^{1+\alpha}, \quad x \in \Omega, t>0, \\
u(x, 0)=u_{0}(x), \quad x \in \Omega, \\
\left.u(x, t)\right|_{\partial \Omega}=0, \quad x \in \partial \Omega, t>0,
\end{gathered}
$$

where $\Omega \subset \mathrm{R}^{n}$ is a bounded domain, $\lambda(s): \mathrm{R}_{+} \rightarrow \mathrm{R}$ is a given continuous function, $\beta, \gamma, \delta,>0$, and $\operatorname{div}\left(|\nabla u|^{p-2} \nabla u\right)$ is the so-called $p$-Laplace operator. By using the concavity method first introduced by Levine [5], under negative initial energy and suitable conditions on $p, \alpha$, and the relaxation function $\lambda(t)$, we prove that there exists finite-time blow-up solution.

Without loss of generality, we choose $\beta=\gamma=\delta=1$ in the following discussion.

\section{Preliminaries and Main Results}

In this section, we introduce some notations, basic definitions, and important lemmas which will be needed in this paper.

For functions $u(x, t), v(x, t)$ defined on $\Omega$, we introduce

$$
\begin{gathered}
(u, v)=\int_{\Omega} u v d x, \quad\|u\|_{2}=\left(\int_{\Omega}|u|^{2} d x\right)^{1 / 2}, \\
\|u\|_{p}=\left(\int_{\Omega}|u|^{p} d x\right)^{1 / p}, \quad\|u\|_{H^{m}}=\left(\sum_{|\alpha| \leq m}\left\|D^{\alpha} u\right\|_{2}^{2}\right)^{1 / 2}, \\
\|u\|_{\infty}=\operatorname{ess} \sup _{x \in \Omega}|u(x)|, \\
\Delta=\frac{\partial^{2}}{\partial x_{1}^{2}}+\cdots+\frac{\partial^{2}}{\partial x_{n}^{2}}, \quad \nabla=\left(\frac{\partial}{\partial x_{1}}, \ldots, \frac{\partial}{\partial x_{n}}\right) .
\end{gathered}
$$


We now construct a space of functions as follows. Let $H^{m}(\Omega)$ denote the Sobolev space with the norm $\|u\|_{H^{m}}=$ $\left(\sum_{|\alpha| \leq m}\left\|D^{\alpha} u\right\|_{2}^{2}\right)^{1 / 2} \cdot C_{0}^{\infty}(\Omega)$ denotes the class of $C^{\infty}$ functions with the compact support in $\Omega . H_{0}^{m}(\Omega)$ denotes the closure in $H^{m}(\Omega)$ of $C_{0}^{\infty}(\Omega)$. The Hilbert space $H_{0}^{m}(\Omega)$ is a subspace of the Sobolev space $H^{m}(\Omega)$.

The following are the basic hypotheses to establish the main results of this paper:

(a) $2<p \leq 2+\alpha<\infty$;

(b) $\lambda$ is a $C^{1}$ function satisfying

$$
\begin{gathered}
\lambda(\tau) \geq 0, \quad \lambda^{\prime}(\tau) \leq 0, \\
\int_{0}^{\infty} \lambda(\tau) d \tau<\frac{\alpha(\alpha+2)}{\alpha(\alpha+2)+1} .
\end{gathered}
$$

To obtain the results of this paper, we will introduce the "modified" energy function:

$$
\begin{aligned}
E(t)= & \frac{1}{2}(\lambda \circ \nabla u)(t) \\
& +\frac{1}{2}\left(1-\int_{0}^{t} \lambda(\tau) d \tau\right)\|\nabla u(t)\|_{2}^{2}+\frac{1}{p}\|\nabla u(t)\|_{p}^{p} \\
& -\frac{1}{2+\alpha} \int_{\Omega} u^{2+\alpha} d x
\end{aligned}
$$

where

$$
(\lambda \circ v)(t)=\int_{0}^{t} \lambda(t-\tau)\|v(t)-v(\tau)\|_{2}^{2} d \tau
$$

The following lemma is similar to the lemma of [21] with slight modification.

Lemma 1. Assume that (10) hold. Let p satisfy (a) and let $u$ be a solution of (8). Then $E(t)$ is nonincreasing function; that is

$$
E^{\prime}(t) \leq 0
$$

Moreover, the following energy inequality holds:

$$
E(t)+\int_{0}^{t}\left\|u_{t}(\tau)\right\|_{H^{1}}^{2} d \tau<E(0) .
$$

Proof. By multiplying the equation in (8) by $u_{t}$, integrating over $\Omega$, we obtain

$$
\begin{aligned}
& \frac{d}{d t}\left[\int_{0}^{t}\left\|u_{t}(\tau)\right\|_{2}^{2} d \tau+\int_{0}^{t}\left\|\nabla u_{t}(\tau)\right\|_{2}^{2} d \tau+\frac{1}{2} \int_{\Omega}|\nabla u|^{2} d x\right. \\
& \left.\quad+\frac{1}{p}\|\nabla u\|_{p}^{p}-\frac{1}{2+\alpha} \int_{\Omega} u^{2+\alpha} d x\right] \\
& \quad-\int_{0}^{t} \lambda(t-\tau) \int_{\Omega} \nabla u_{t}(t) \nabla u(\tau) d x d \tau=0 .
\end{aligned}
$$

For the last term on the left side of (16),

$$
\begin{aligned}
& \int_{0}^{t} \lambda(t-\tau) \int_{\Omega} \nabla u_{t}(t) \nabla u(\tau) d x d \tau \\
& =\int_{0}^{t} \lambda(t-\tau) \int_{\Omega} \nabla u_{t}(t)[\nabla u(\tau)-\nabla u(t)] d x d \tau \\
& \quad+\int_{0}^{t} \lambda(t-\tau) \int_{\Omega} \nabla u_{t}(t) \nabla u(t) d x d \tau \\
& =-\frac{1}{2} \int_{0}^{t} \lambda(t-\tau)\left[\frac{d}{d t} \int_{\Omega}|\nabla u(\tau)-\nabla u(t)|^{2} d x\right] d \tau \\
& \quad+\frac{1}{2} \int_{0}^{t} \lambda(\tau)\left[\frac{d}{d t} \int_{\Omega}|\nabla u(t)|^{2} d x\right] d \tau \\
& =-\frac{1}{2} \frac{d}{d t}\left[\int_{0}^{t} \lambda(t-\tau) \int_{\Omega}|\nabla u(\tau)-\nabla u(t)|^{2} d x d \tau\right] \\
& \quad+\frac{1}{2} \frac{d}{d t}\left[\int_{0}^{t} \lambda(\tau) \int_{\Omega}|\nabla u(t)|^{2} d x d \tau\right] \\
& \quad+\frac{1}{2} \int_{0}^{t} \lambda^{\prime}(t-\tau) \int_{\Omega}|\nabla u(\tau)-\nabla u(t)|^{2} d x d \tau \\
& \quad-\frac{1}{2} \lambda(t) \int_{\Omega}|\nabla u(t)|^{2} d x .
\end{aligned}
$$

Inserting (17) into (16), we have

$$
\begin{aligned}
& \frac{d}{d t}\left[\int_{0}^{t}\left\|u_{t}(\tau)\right\|_{2}^{2} d \tau+\int_{0}^{t}\left\|\nabla u_{t}(\tau)\right\|_{2}^{2} d \tau+\frac{1}{2} \int_{\Omega}|\nabla u|^{2} d x\right. \\
& \left.\quad+\frac{1}{p}\|\nabla u\|_{p}^{p}-\frac{1}{2+\alpha} \int_{\Omega} u^{2+\alpha} d x\right] \\
& \quad+\frac{1}{2} \frac{d}{d t}\left[\int_{0}^{t} \lambda(t-\tau) \int_{\Omega}|\nabla u(\tau)-\nabla u(t)|^{2} d x d \tau\right] \\
& \quad-\frac{1}{2} \frac{d}{d t}\left[\int_{0}^{t} \lambda(\tau) \int_{\Omega}|\nabla u(t)|^{2} d x d \tau\right] \\
& =\frac{1}{2} \int_{0}^{t} \lambda^{\prime}(t-\tau) \int_{\Omega}|\nabla u(\tau)-\nabla u(t)|^{2} d x d \tau \\
& \quad-\frac{1}{2} \lambda(t) \int_{\Omega}|\nabla u(t)|^{2} d x \leq 0,
\end{aligned}
$$

for regular solution. The proof of Lemma 1 is completed. This result is valid for weak solutions by a simple density argument.

Now we consider the finite time blow-up of solutions with $E(0)<0$ for the problem (8).

Theorem 2. Let $p$ satisfy (a) and let the relaxation function $\lambda(s)$ be a $C^{1}$ function satisfying (10) and (11). Assume that $u_{0} \in H_{0}^{1}(\Omega)$ such that $E(0)<0$. Then the solutions $u(x, t)$ of the problem (8) blow up in finite time; that is, the maximum existence time $T_{\max }$ of $u(x, t)$ is finite and

$$
\lim _{t \mapsto T_{\max }}\|u(x, t)\|_{H^{1}}^{2}=+\infty .
$$


Proof. The proof makes use of the so-called "concavity" arguments. For any $T_{0}>0$, let

$$
\begin{aligned}
M(t)= & \int_{0}^{t}\|u(\tau)\|_{2}^{2} d \tau \\
& +\int_{0}^{t}\|\nabla u(\tau)\|_{2}^{2} d \tau+\left(T_{0}-t\right)\left(\left\|\nabla u_{0}\right\|_{2}^{2}+\left\|u_{0}\right\|_{2}^{2}\right),
\end{aligned}
$$

for $t \in\left[0, T_{0}\right]$.

A direct computation yields

$$
\begin{aligned}
M^{\prime}(t)= & \|u(t)\|_{2}^{2}+\|\nabla u(t)\|_{2}^{2} \\
& -\left(\left\|\nabla u_{0}\right\|_{2}^{2}+\left\|u_{0}\right\|_{2}^{2}\right) \\
= & 2 \int_{0}^{t}\left(u, u_{t}\right) d \tau+2 \int_{0}^{t}\left(\nabla u, \nabla u_{t}\right) d \tau, \\
M^{\prime \prime}(t)= & 2\left(u, u_{t}\right)+2\left(\nabla u, \nabla u_{t}\right) .
\end{aligned}
$$

By multiplying (8) with $u$ and integrating over $\Omega$,

$$
\begin{aligned}
& \left(u, u_{t}\right)+\left(\nabla u, \nabla u_{t}\right) \\
& =-\|\nabla u(t)\|_{2}^{2}-\int_{\Omega} \int_{0}^{t} \lambda(t-\tau) \Delta u(\tau) d \tau u(t) d x \\
& \quad-\|\nabla u(t)\|_{p}^{p}+\int_{\Omega} u^{2+\alpha} d x .
\end{aligned}
$$

This implies that

$$
\begin{aligned}
M^{\prime \prime}(t)= & -2\|\nabla u(t)\|_{2}^{2} \\
& -2 \int_{\Omega} \int_{0}^{t} \lambda(t-\tau) \Delta u(\tau) d \tau u(t) d x \\
& -2\|\nabla u(t)\|_{p}^{p}+\int_{\Omega} u^{2+\alpha} d x
\end{aligned}
$$

and we have

$$
\begin{aligned}
& M^{\prime \prime}(t) M(t)-\frac{\alpha+4}{4} M^{\prime}(t)^{2} \\
& =2 M(t)\left[-\|\nabla u(t)\|_{2}^{2}-\int_{\Omega} \int_{0}^{t} \lambda(t-\tau) \Delta u(\tau) d \tau u(t) d x\right. \\
& \left.\quad-\|\nabla u(t)\|_{p}^{p}+\int_{\Omega} u^{2+\alpha} d x\right] \\
& -\frac{\alpha+4}{4}\left[2 \int_{0}^{t}\left(u, u_{t}\right) d \tau+2 \int_{0}^{t}\left(\nabla u, \nabla u_{t}\right) d \tau\right]^{2} \\
& =2 M(t)\left[-\|\nabla u(t)\|_{2}^{2}-\int_{\Omega} \int_{0}^{t} \lambda(t-\tau) \Delta u(\tau) d \tau u(t) d x\right.
\end{aligned}
$$

$$
\begin{gathered}
\left.-\|\nabla u(t)\|_{p}^{p}+\int_{\Omega} u^{2+\alpha} d x\right]+(\alpha+4) \\
\times\left\{H(t)-\left[M(t)-\left(T_{0}-t\right)\left(\left\|\nabla u_{0}\right\|_{2}^{2}+\left\|u_{0}\right\|_{2}^{2}\right)\right]\right. \\
\left.\times\left[\int_{0}^{t}\left(u_{t}, u_{t}\right) d \tau+\int_{0}^{t}\left(\nabla u_{t}, \nabla u_{t}\right) d \tau\right]\right\},
\end{gathered}
$$

where

$$
\begin{aligned}
H(t)= & {\left[\int_{0}^{t}(u, u) d \tau+\int_{0}^{t}(\nabla u, \nabla u) d \tau\right] } \\
& \times\left[\int_{0}^{t}\left(u_{t}, u_{t}\right) d \tau+\int_{0}^{t}\left(\nabla u_{t}, \nabla u_{t}\right) d \tau\right] \\
& -\left[\int_{0}^{t}\left(u, u_{t}\right) d \tau+\int_{0}^{t}\left(\nabla u, \nabla u_{t}\right) d \tau\right]^{2}
\end{aligned}
$$

Using Schwartz's inequality, we have

$$
\begin{aligned}
& \left(\int_{0}^{t}\left(u, u_{t}\right) d \tau\right)^{2} \leq \int_{0}^{t}\|u\|_{2}^{2} d \tau \int_{0}^{t}\left\|u_{t}\right\|_{2}^{2} d \tau \\
& \left(\int_{0}^{t}\left(\nabla u, \nabla u_{t}\right) d \tau\right)^{2} \leq \int_{0}^{t}\|\nabla u\|_{2}^{2} d \tau \int_{0}^{t}\left\|\nabla u_{t}\right\|_{2}^{2} d \tau \\
& \int_{0}^{t}\left(u, u_{t}\right) d \tau \int_{0}^{t}\left(\nabla u, \nabla u_{t}\right) d \tau \\
& \leq \frac{1}{2} \int_{0}^{t}\|u\|_{2}^{2} d \tau \int_{0}^{t}\left\|\nabla u_{t}\right\|_{2}^{2} d \tau \\
& \quad+\frac{1}{2} \int_{0}^{t}\left\|u_{t}\right\|_{2}^{2} d \tau \int_{0}^{t}\|\nabla u\|_{2}^{2} d \tau
\end{aligned}
$$

By (26), we have

$$
H(t) \geq 0, \quad t \in\left[0, T_{0}\right] .
$$

Thus,

$$
M^{\prime \prime}(t) M(t)-\frac{\alpha+4}{4} M^{\prime}(t)^{2} \geq M(t) \eta(t),
$$

where

$$
\begin{aligned}
\eta(t)= & -(\alpha+4)\left[\int_{0}^{t}\left(u_{t}, u_{t}\right) d \tau+\int_{0}^{t}\left(\nabla u_{t}, \nabla u_{t}\right) d \tau\right] \\
& -2\|\nabla u(t)\|_{2}^{2}-2 \int_{\Omega} \int_{0}^{t} \lambda(t-\tau) \Delta u(\tau) d \tau u(t) d x \\
& -2\|\nabla u(t)\|_{p}^{p}+2 \int_{\Omega} u^{2+\alpha} d x .
\end{aligned}
$$


For the third term on the left of (29), we have

$$
\begin{aligned}
& -\int_{\Omega} \int_{0}^{t} \lambda(t-\tau) \Delta u(\tau) d \tau u(t) d x \\
& =\int_{0}^{t} \lambda(t-\tau) \int_{\Omega} \nabla u(\tau) \nabla u(t) d x d \tau \\
& =\int_{0}^{t} \lambda(t-\tau) \int_{\Omega} \nabla u(t) \nabla[u(\tau)-u(t)] d x d \tau \\
& \quad+\int_{0}^{t} \lambda(t-\tau)\|\nabla u(t)\|_{2}^{2} d \tau .
\end{aligned}
$$

By (29) and (30), we have

$$
\begin{aligned}
& \eta(t)=-(\alpha+4) \int_{0}^{t}\left\|u_{t}\right\|_{H^{1}}^{2} d \tau \\
& -2\left(1-\int_{0}^{t} \lambda(t-\tau) d \tau\right)\|\nabla u(t)\|_{2}^{2} \\
& +2 \int_{0}^{t} \lambda(t-\tau) \int_{\Omega} \nabla u(t)[\nabla u(\tau)-\nabla u(t)] d x d \tau \\
& -2\|\nabla u\|_{p}^{p}+2 \int_{\Omega} u^{2+\alpha} d x \\
& \geq-(\alpha+4) \int_{0}^{t}\left\|u_{t}\right\|_{H^{1}}^{2} d \tau \\
& -2\left(1-\int_{0}^{t} \lambda(t-\tau) d \tau\right)\|\nabla u(t)\|_{2}^{2}-2\|\nabla u\|_{p}^{p} \\
& +2 \int_{\Omega} u^{2+\alpha} d x \\
& -2\left[\frac{\alpha+2}{2} \int_{0}^{t} \lambda(t-\tau)\|\nabla u(\tau)-\nabla u(t)\|_{2}^{2} d \tau\right. \\
& \left.+\frac{1}{2(\alpha+2)} \int_{0}^{t} \lambda(t-\tau)\|\nabla u(t)\|_{2}^{2} d \tau\right] \\
& =-2(\alpha+2) E(t)+(\alpha+2)\left(1-\int_{0}^{t} \lambda(t-\tau) d \tau\right) \\
& \times\|\nabla u(t)\|_{2}^{2}+\frac{2(\alpha+2)}{p}\|\nabla u\|_{p}^{p} \\
& -(\alpha+4) \int_{0}^{t}\left\|u_{t}\right\|_{H^{1}}^{2} d \tau-\frac{1}{\alpha+2} \int_{0}^{t} \lambda(\tau) d \tau\|\nabla u(t)\|_{2}^{2} \\
& -2\left(1-\int_{0}^{t} \lambda(t-\tau) d \tau\right)\|\nabla u(t)\|_{2}^{2}-2\|\nabla u\|_{p}^{p} \\
& =-2(\alpha+2) E(t)+\alpha\left(1-\int_{0}^{t} \lambda(t-\tau) d \tau\right)\|\nabla u(t)\|_{2}^{2} \\
& -\frac{1}{\alpha+2} \int_{0}^{t} \lambda(\tau) d \tau\|\nabla u(t)\|_{2}^{2} \\
& -(\alpha+4) \int_{0}^{t}\left\|u_{t}\right\|_{H^{1}}^{2} d \tau+\frac{2 \alpha-2 p+4}{p}\|\nabla u\|_{p}^{p} .
\end{aligned}
$$

Using Lemma 1, we have

$$
E(t)+\int_{0}^{t}\left\|u_{t}\right\|_{H^{1}}^{2} d \tau<E(0)
$$

and then

$$
\begin{aligned}
\eta(t) \geq & -2(\alpha+2) E(0)+\alpha \int_{0}^{t}\left\|u_{t}\right\|_{H^{1}}^{2} d \tau \\
& +\left[\alpha\left(1-\int_{0}^{t} \lambda(\tau) d \tau\right)-\frac{1}{\alpha+2} \int_{0}^{t} \lambda(\tau) d \tau\right]\|\nabla u(t)\|_{2}^{2} \\
= & -2(\alpha+2) E(0)+\alpha \int_{0}^{t}\left\|u_{t}\right\|_{H^{1}}^{2} d \tau \\
& +\left[\alpha-\frac{\alpha(\alpha+2)+1}{\alpha+2} \int_{0}^{t} \lambda(\tau) d \tau\right]\|\nabla u(t)\|_{2}^{2} .
\end{aligned}
$$

Since

$$
\int_{0}^{\infty} \lambda(\tau) d \tau<\frac{\alpha(\alpha+2)}{\alpha(\alpha+2)+1}
$$

this implies that

$$
\eta(t)>\delta, \quad 0 \leq t<\infty
$$

where $\delta$ is a positive constant.

From the discussion above, we see that

$$
M^{\prime \prime}(t) M(t)-\frac{\alpha+4}{4} M^{\prime}(t)^{2} \geq M(t) \delta .
$$

From the definition of $M(t)$, there exists $\rho>0$, such that

$$
M(t) \geq \rho, \quad \text { for } t \in[0, T),
$$

and we have

$$
M^{\prime \prime}(t) M(t)-\frac{\alpha+4}{4} M^{\prime}(t)^{2} \geq \rho \delta .
$$

Thus,

$$
\begin{aligned}
& \left(M(t)^{-\alpha / 4}\right)^{\prime \prime} \\
& \quad=\left(-\frac{\alpha}{4}\right) M(t)^{-\alpha / 4-2}\left[M^{\prime \prime}(t) M(t)-\frac{\alpha+4}{4} M^{\prime}(t)^{2}\right] \\
& \quad \leq\left(-\frac{\alpha}{4}\right) \rho \delta M(T)^{-(\alpha+8) / 4}<0 .
\end{aligned}
$$

Hence, this proves that $M(t)^{-\alpha / 4}$ reaches 0 in finite time as $t \rightarrow T_{1}^{-}$. Since $T_{1}$ is independent of $T$, we may assume that $T_{1}<T$.

This means

$$
\lim _{t \rightarrow T_{1}^{-}} M(t)=+\infty
$$


or

$$
\begin{aligned}
\lim _{t \rightarrow T_{1}^{-}}\left(\int_{0}^{t}\|u(\tau)\|_{2}^{2} d \tau+\int_{0}^{t}\|\nabla u(\tau)\|_{2}^{2} d \tau\right. \\
\left.\quad+\left(T_{0}-t\right)\left(\left\|\nabla u_{0}\right\|_{2}^{2}+\left\|u_{0}\right\|_{2}^{2}\right)\right)=+\infty
\end{aligned}
$$

This implies that

$$
\lim _{t \rightarrow T_{1}^{-}} \int_{0}^{t}\|u(\tau)\|_{H_{1}}^{2} d \tau=+\infty .
$$

Then, the desired assertion immediately follows.

Remark 1. In the absence of the viscous term $\left(\Delta u_{t}\right)$ or $p$ Laplace operator $\left(\operatorname{div}\left(|\nabla u|^{p-2} \nabla u\right)\right)$ for the problem (8), the equation reduced to

$$
\begin{aligned}
& \text { (1) } u_{t}-\Delta u+\int_{0}^{t} \lambda(t-\tau) \Delta u(\tau) d \tau=u^{1+\alpha} \\
& \text { (2) } u_{t}-\Delta u-\Delta u_{t}+\int_{0}^{t} \lambda(t-\tau) \Delta u(\tau) d \tau=u^{1+\alpha} \\
& \text { (3) } u_{t}-\Delta u+\int_{0}^{t} \lambda(t-\tau) \Delta u(\tau) d \tau=\operatorname{div}\left(|\nabla u|^{p-2} \nabla u\right)+u^{1+\alpha}
\end{aligned}
$$

and from the process of the proof, we can see that the results of Theorem 2 still hold.

\section{Conflict of Interests}

The authors declare that there is no conflict of interests regarding the publication of this paper.

\section{Acknowledgments}

This work is supported by the NSF of China (40890150 and 40890153) and the Scientific Program (2008B080701042) of Guangdong Province. The authors thank the anonymous referees for their helpful comments and suggestions.

\section{References}

[1] A. B. Al'shin, M. O. Korpusov, and A. G. Sveshnikov, Sveshnikov, Blow-up in Nonlinear Sobolev Type Equations, De Gruyter, 2011.

[2] A. Y. Kolesov, E. F. Mishchenko, and N. K. Rozov, "Asymptotic methods for studying periodic solutions of nonlinear hyperbolic equations," Trudy Matematicheskogo Instituta Imeni V. A. Steklova, vol. 222, pp. 3-198, 1998.

[3] B. K. Shivamoggi, "A symmetric regularized long-wave equation for shallow water waves," The Physics of Fluids, vol. 29, no. 3, pp. 890-891, 1986.

[4] P. Rosenau, "Evolution and breaking of the ion-acoustic waves," Physics of Fluids, vol. 31, no. 6, pp. 1317-1319, 1988.

[5] H. A. Levine, "Some nonexistence and instability theorems for solutions of formally parabolic equations of the form $P u_{t}=$ $-A u+f(u), "$ Archive for Rational Mechanics and Analysis, vol. 51, pp. 371-386, 1973.

[6] M. X. Wang and X. Q. Ding, "Global solutions, asymptotic behavior and blow-up problems for semilinear heat equations," Science in China A, vol. 35, pp. 1026-1034, 1992.
[7] F. Gazzola and T. Weth, "Finite time blow-up and global solutions for semilinear parabolic equations with initial data at high energy level," Differential and Integral Equations, vol. 18, no. 9, pp. 961-990, 2005.

[8] Y. C. Liu, R. Z. Xu, and T. Yu, "Global existence, nonexistence and asymptotic behavior of solutions for the Cauchy problem of semilinear heat equations," Nonlinear Analysis: Theory, Methods \& Applications, vol. 68, no. 11, pp. 3332-3348, 2008.

[9] H. Wang and Y. He, "On blow-up of solutions for a semilinear parabolic equation involving variable source and positive initial energy," Applied Mathematics Letters, vol. 26, no. 10, pp. 10081012, 2013.

[10] E. I. Kaikina, P. I. Naumkin, and I. Shishmarev, "A periodic problem for a Sobolev-type nonlinear equation," Function Analysis and Its Applications, vol. 44, no. 3, pp. 14-26, 2010.

[11] Y. Cao, J. X. Yin, and C. H. Jin, "A periodic problem of a semilinear pseudoparabolic equation," Abstract and Applied Analysis, vol. 2011, Article ID 363579, 27 pages, 2011.

[12] Y. Cao, J. Yin, and C. Wang, "Cauchy problems of semilinear pseudo-parabolic equations," Journal of Differential Equations, vol. 246, no. 12, pp. 4568-4590, 2009.

[13] E. I. Kaikina, "Initial boundary value problems for nonlinear pseudo-parabolic equations in a critical case," Electronic Journal of Differential Equation, vol. 109, pp. 1-25, 2007.

[14] R. Xu and J. Su, "Global existence and finite time blow-up for a class of semilinear pseudo-parabolic equations," Journal of Functional Analysis, vol. 264, no. 12, pp. 2732-2763, 2013.

[15] M. Tsutsumi, "Existence and nonexistence of global solutions for nonlinear parabolic equations," Publications of the Research Institute for Mathematical Sciences, vol. 8, no. 2, pp. 211-229, 1972.

[16] Y. C. Liu and J. S. Zhao, "Nonlinear parabolic equations with critical initial conditions $J_{\left(u_{0}\right)}=d$ or $I_{\left(u_{0}\right)}=0$," Nonlinear Analysis: Theory, Methods \& Applications, vol. 58, no. 7-8, pp. 873-883, 2004.

[17] R. Xu, X. Cao, and T. Yu, "Finite time blow-up and global solutions for a class of semilinear parabolic equations at high energy level," Nonlinear Analysis: Real World Applications, vol. 13, no. 1, pp. 197-202, 2012.

[18] S. A. Messaoudi, "A note on blow up of solutions of a quasilinear heat equation with vanishing initial energy," Journal of Mathematical Analysis and Applications, vol. 273, no. 1, pp. 243-247, 2002.

[19] G. Gripenberg, "Global existence of solutions of Volterra integrodifferential equations of parabolic type," Journal of Differential Equations, vol. 102, no. 2, pp. 382-390, 1993.

[20] H. Yin, "Weak and classical solutions of some nonlinear volterra integrodifferential equations," Communications in Partial Differential Equations, vol. 17, no. 7-8, pp. 1369-1385, 1992.

[21] S. A. Messaoudi, "Blow-up of solutions of a semi linear heat equation with a Visco-elastic term," in Nonlinear Elliptic and Parabolic Problems, H. Brezis, M. Chipot, and J. Escher, Eds., vol. 64 of Progress in Nonlinear Differential Equations and Their Applications, pp. 351-356, Birkhäuser, Basel, Switzerland, 2005.

[22] Y. D. Shang and B. L. Guo, "On the problem of the existence of global solutions for a class of nonlinear convolutional integro-differential equations of pseudoparabolic type," Acta Mathematicae Applicatae Sinica, vol. 26, no. 3, pp. 512-524, 2003.

[23] Y. D. Shang and B. L. Guo, "Initial boundary value problem and initial value problem for the nonlinear integro-differential equations of pseudoparabolic type," Mathematica Applicata, vol. 15 , no. 1, pp. 40-45, 2002. 
[24] Y. D. Shang and B. L. Guo, "Initial-boundary value problems for a class of quasilinear integro-differential equations of pseudoparabolic type," Chinese Journal of Engineering Mathematics, vol. 20, no. 4, pp. 1-6, 2003.

[25] M. Ptashnyk, "Degenerate quaslinear pseudoparabolic equations with memory terms and variational inequalities," Nonlinear Analysis: Theory, Methods \& Applications, vol. 66, no. 12, pp. 2653-2675, 2007. 


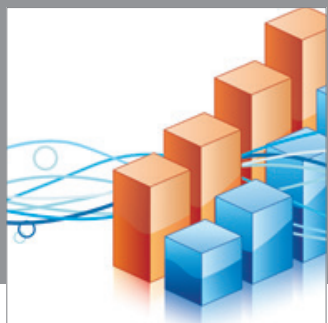

Advances in

Operations Research

mansans

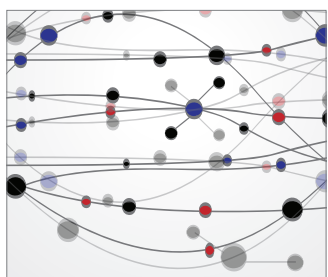

The Scientific World Journal
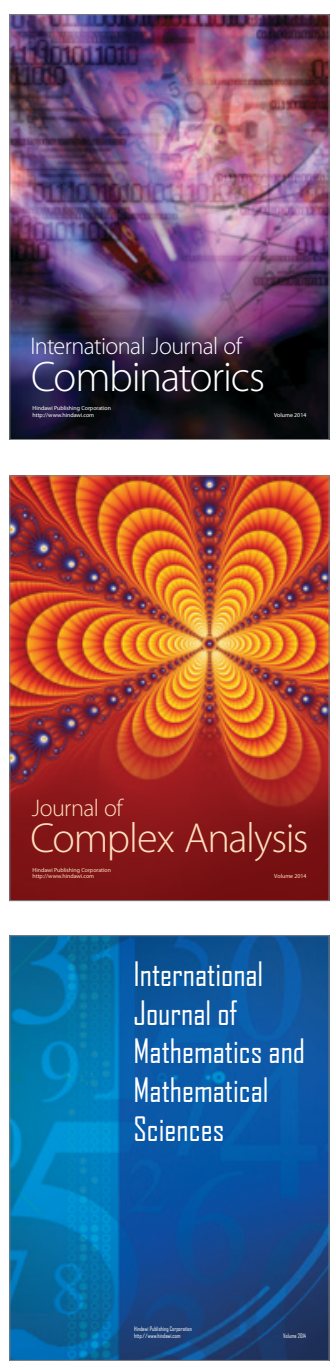
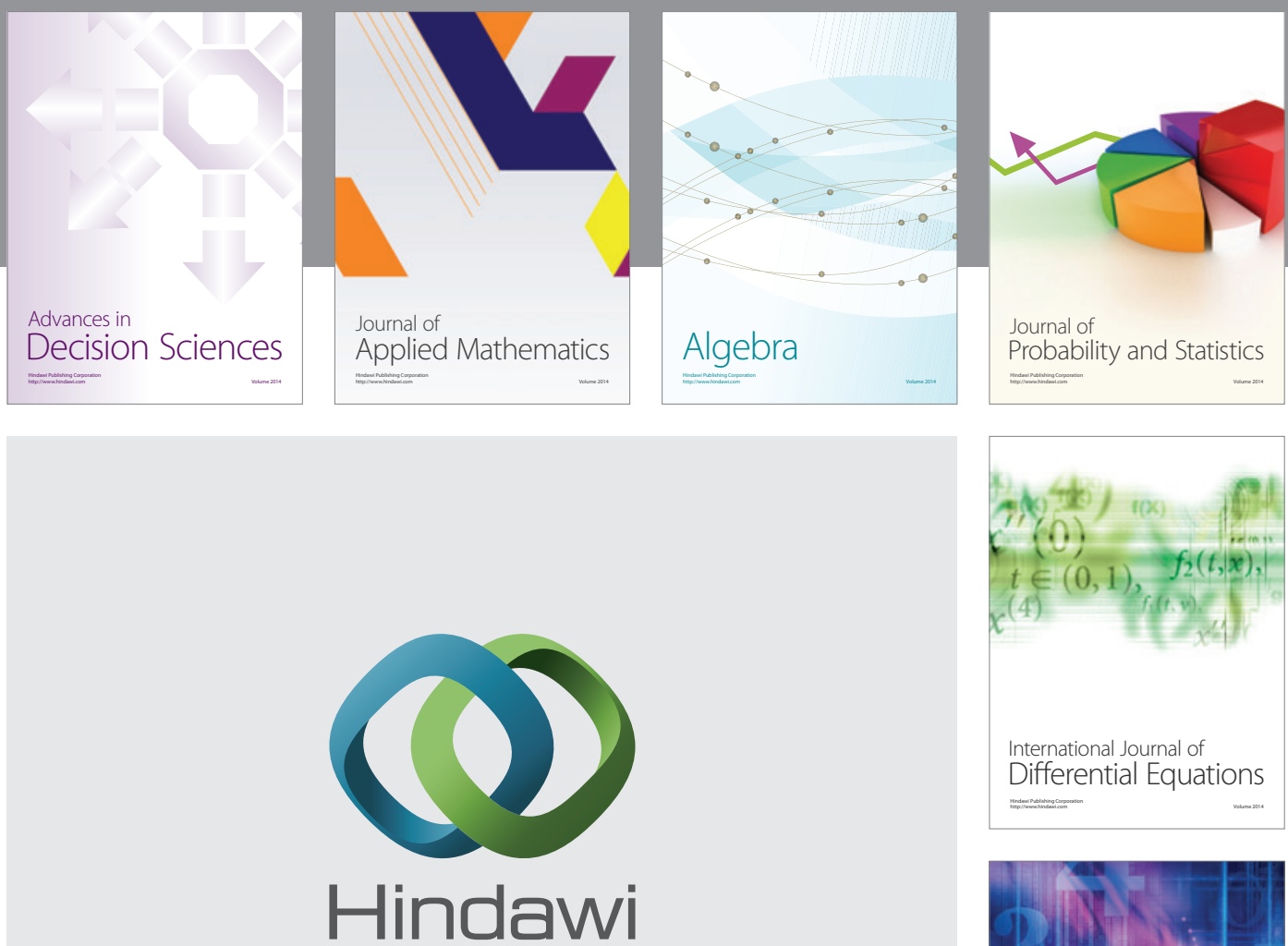

Submit your manuscripts at http://www.hindawi.com
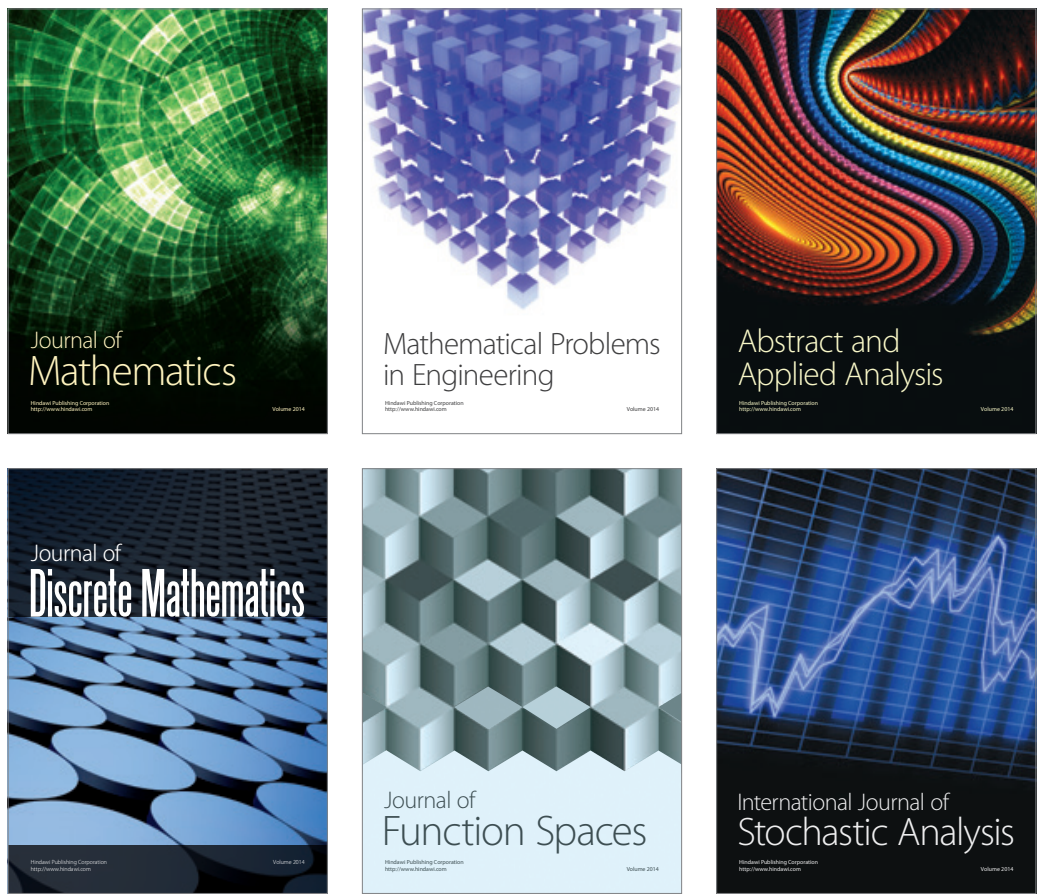

Journal of

Function Spaces

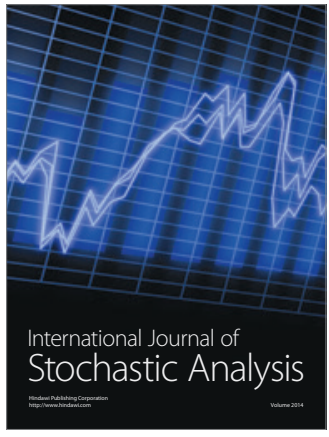

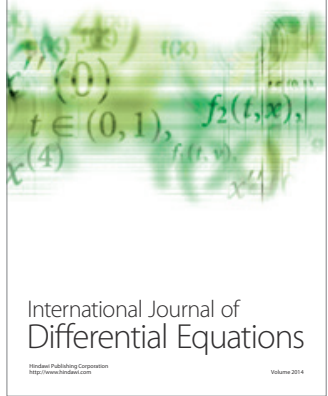
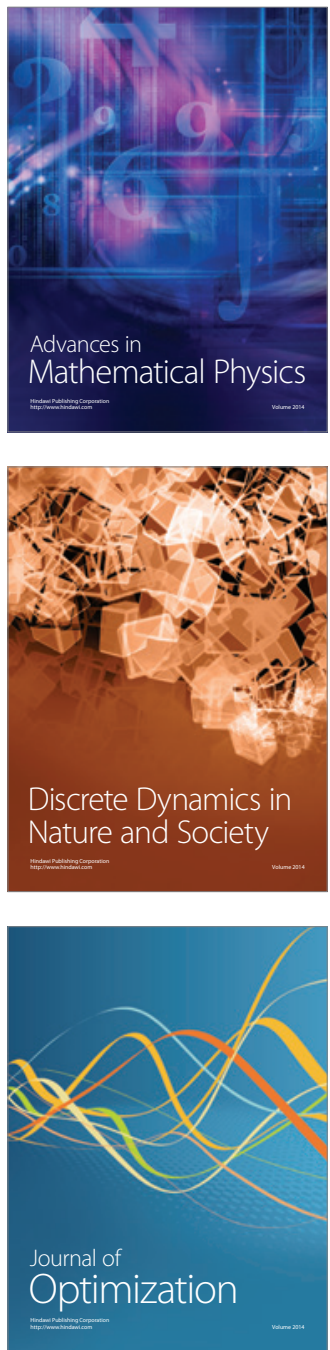